\title{
A SIMULATION OF THE RESONATOR HOSE USING METHOD OF CHARACTERISTICS
}

\author{
Kiyokazu NAGATA $^{*}$, Koji TAKAHASHI ${ }^{* *}$ \\ Basic Technology Research and Development Center \\ Kayaba Industry Co., Ltd. \\ 1-12-1, Asamizodai, Sagamihara-shi, Kanagawa 228, Japan \\ Department of Mechanical Engineering, Faculty of Science \\ Sophia University \\ 7-1, Kioicho, Chiyoda-ku, Tokyo 102, Japan
}

\begin{abstract}
This paper describes a simulation technique for resonator hoses which are used in the hydraulic systems of automobiles. In order to calculate the characteristics of resonator hose in a circuit, a distributed parameter model considering the effect of the leakage through the wall of the inner tube has been developed. In calculation, the method of characteristics was employed to take into account the non-linearity of the leakage. The validity of the model was confirmed by experimental results. Furthermore, the flow characteristics of the inner tube of the resonator hoses were tested to obtain better simulated results. Using this simulation model, the effect of parameters such as leakage coefficient, length of the inner tube, and pressure loss in the inner tube were investigated.
\end{abstract}

\section{KEY WORDS}

Simulation, Resonator hose, Method of characteristics, Co-axial pipe, Leakage

\section{NOMENCLATURE}

$A$ : Cross sectional area.

$a$ : Wave speed.

$d$ : Damping coefficient of the outer-tube wall model.

$f$ : Pressure loss in the tube per unit length.

$f_{l}$ :First-order frequency of the harmonics of the ripple.

$K$ : Bulk modulus of the fluid. $k_{l}, k_{2}$ : Spring stiffness of the outer-tube wall model.

$k_{h}$ : Spring stiffness of the fluid in the outer tube.

$k_{T}$ : Series combination of $k_{l}$ and $k_{h}$

$L$ : Length of the tube.

$P$ : Pressure.

$Q_{L}$ : Leakage from the inner tube per unit length.

$R$ : Leakage coefficient.

$r$ : Inner radius of the tube 


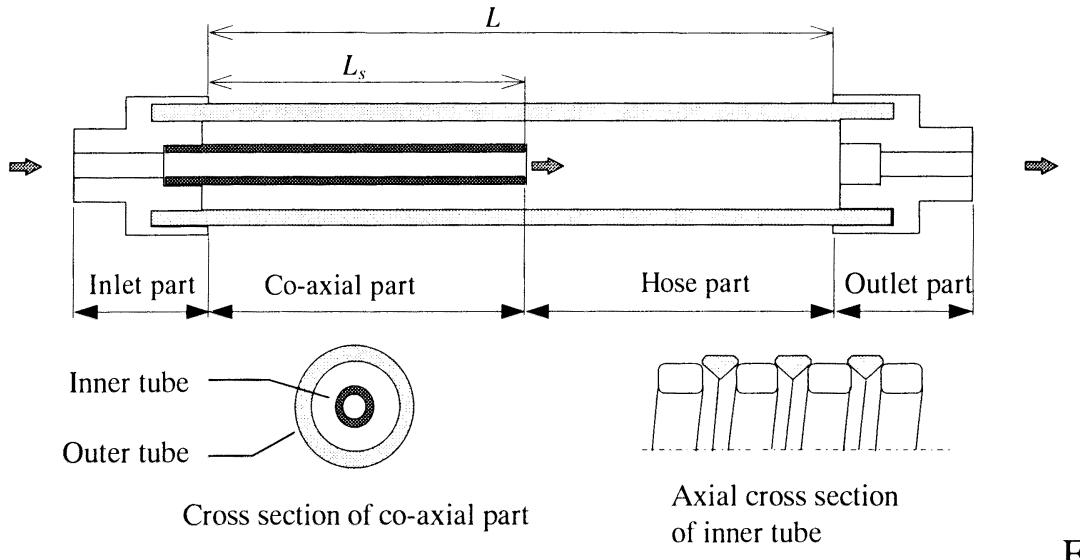

Figure 1. Structure of a resonator hose

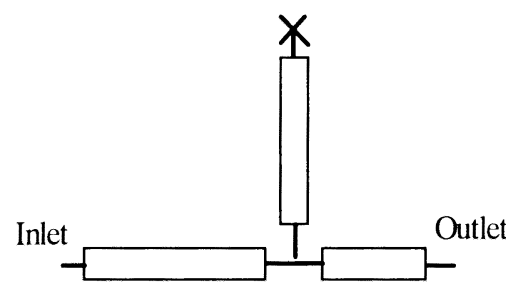

Figure 2. Schematic diagram of a conventional model $t$ : Time.

$V$ : Velocity.

$x$ : Space coordinate along the tube.

$y$ : Displacement of the outer-tube wall.

$\alpha$ : Modification factor.

$\rho$ : Density of the fluid.

Subscripts

$h$ : Hose

$i$ : Inner tube

in: input

$o$ : Outer tube

out: output

\section{INTRODUCTION}

In the hydraulic circuit of an automobile such as a power-steering system of a passenger car, it is important to reduce the fluid borne noise generated by a pump from the viewpoint of keeping the silence in a cabin. Resonator hoses are frequently used in the circuit, because of its effectiveness in the reduction of the pressure ripple from the pump. In the design stage of the circuit, however, the effects of design parameters on the pressure ripple such as the length, diameter, and stiffness of the inner and outer tubes are not clear, and the placing and direction of the resonator hose in a circuit could not be selected theoretically. Therefore it is necessary to construct actual circuits and to investigate them experimentally by trial and errer.

Computer simulation techniques are very useful for avoiding such inefficiency. The most simple modelling means is to regard it as a branch circuit as shown in Figure 2. Using this model, some papers have been reported their simulated results[1],[2]. To calculate this model, they used the frequency domain technique such as impedance method. The frequency domain technique, however, is based on the assumption of the linearity of the model. Therefore, if an element of the system including resonator hoses has any non-linear characteristic, this approach is not suitable. Then the time domain techniques would be suitable to calculate the non-linear characteristic.

We have already developed a hydraulic circuit simulation program 'PAP' using the method of characteristics ( time domain technique ) to predict the dynamic response and pressure ripple in any hydraulic circuits with hoses, pumps and valves[3]. Therefore, it is possible to simulate the circuits which have resonator hoses in time domain by adding its model.

In this paper, a model of resonator hose considering the leakage through the inner tube wall is introduced. To calculate it, method of characteristics is employed. Then the effects of the length and leakage of the inner tube on the hydraulic characteristics are discussed based on the simulated results.

\section{MODELLING OF A RESONATOR HOSE}

A resonator hose mainly consists of the following three parts (Figure 1) ;

1) Inlet and outer parts,

2) Coaxial part, and 


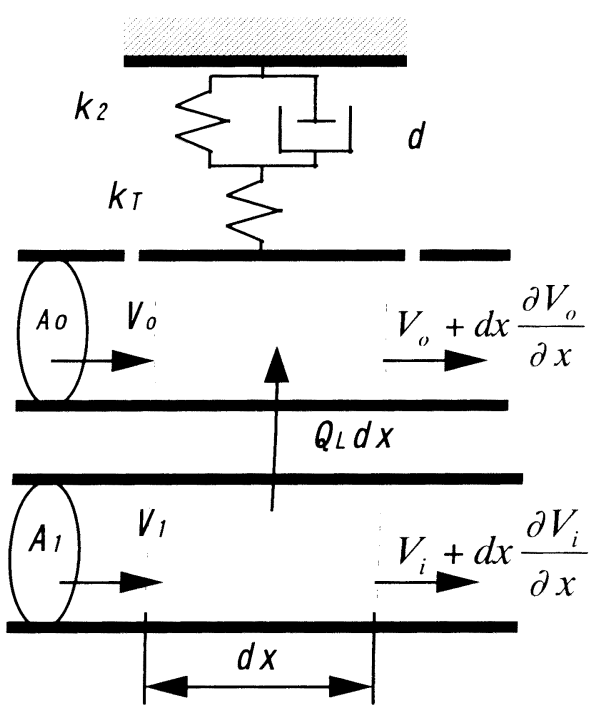

Figure 3. Model of the co-axial part

3) Hose part.

Prior to model these parts, let us assume the following ;

1) The flow is one dimensional.

2) Inlet and outlet parts are regarded as common hydraulic pipeline.

3) Co-axial part can be divided into two pipelines; inner tube and outer tube. The inner-tube wall is regarded as a series of distributed orifices through which the fluid leaks.

4) Interaction between the inner and outer tubes caused by the motion of the inner-tube wall is negligible.

5) Outer-tube wall has a visco-elastic characteristics which can be expressed by two springs and a damper. as shown in Figure 3.

Under these assumptions, basic equations of the co-axial part can be expressed as follows:

$\frac{1}{\rho} \frac{\partial P_{i}}{\partial x}+\frac{\partial V_{i}}{\partial t}+V_{i} \frac{\partial V_{i}}{\partial x}+\frac{\alpha_{i}}{\rho} f_{i}=0$

$\frac{\partial V_{i}}{\partial x}+\frac{1}{K_{i}}\left(\frac{\partial P_{i}}{\partial t}+V_{i} \frac{\partial P_{i}}{\partial x}\right)+\frac{Q_{L}}{A_{i}}=0$

$\frac{1}{\rho} \frac{\partial P_{o}}{\partial x}+\frac{\partial V_{o}}{\partial t}+V_{i} \frac{\partial V_{o}}{\partial x}+\frac{1}{\rho} f_{o}=0$

$\frac{\partial V_{o}}{\partial x}+\frac{2}{r_{o} k_{T}} \frac{\partial P_{o}}{\partial t}+\frac{2}{r_{o} d}\left(1+\frac{k_{2}}{k_{1}}\right) p_{W}-\frac{2 k_{2}}{r_{o} d} y-\frac{Q_{L}}{A_{o}}=0$

The motion of the outer-tube wall is expressed by the following equation:
$\frac{D y}{D t}=\frac{1}{d}\left(1+\frac{k_{2}}{k_{T}}\right) P_{W}+\frac{1}{d} k_{2} y+\frac{1}{k_{2}} \frac{D P_{W}}{D t}$

The leakage through the inner-tube wall is expressed by

$Q_{L}=R \sqrt{\left|P_{i}-P_{o}\right|}$

The pressure losses $f_{i}$ and $f_{o}$ in the tubes include frequency dependent friction terms.

Equations (1), (2), (3), (4), and (5) are transformed to ordinary differential equations by means of the method of characteristics as follows:

For inner region,

$\frac{d x}{d t}= \pm a_{i}$

$\frac{ \pm 1}{\rho a_{i}} \frac{d P_{i}}{d t}+\frac{d V_{i}}{d t}+\frac{Q_{L}}{A_{i}}+\frac{1}{\rho} f_{i}=0$

For outer region,

$\frac{d x}{d t}= \pm a_{o}$

$\frac{ \pm 1}{\rho a_{o}} \frac{d P_{o}}{d t}+\frac{d V_{o}}{d t} \pm \frac{2 a_{o}}{r_{o} d}\left(\left(1+\frac{k_{2}}{k_{T}}\right) P_{o}-k_{2} y\right)$

$-\frac{Q_{L}}{A_{o}}+\frac{1}{\rho} f_{o}=0$

Where,

$a_{i}=\sqrt{\frac{K}{\rho}} \quad(11), \quad a_{o}=\sqrt{\frac{r_{o} k_{T}}{2 \rho}}$

The basic equations for the hose part (outer tube only) are also derived out by similar manner omitting the leakage term and using different friction term.

To connect these parts, continuity equations are applied.

\section{MODELLING OF CIRCUITS}

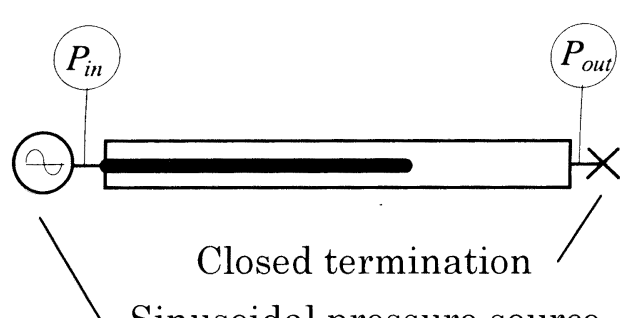

Sinusoidal pressure source

Figure 4. Schematic diagram of the resonator-hose test circuit 


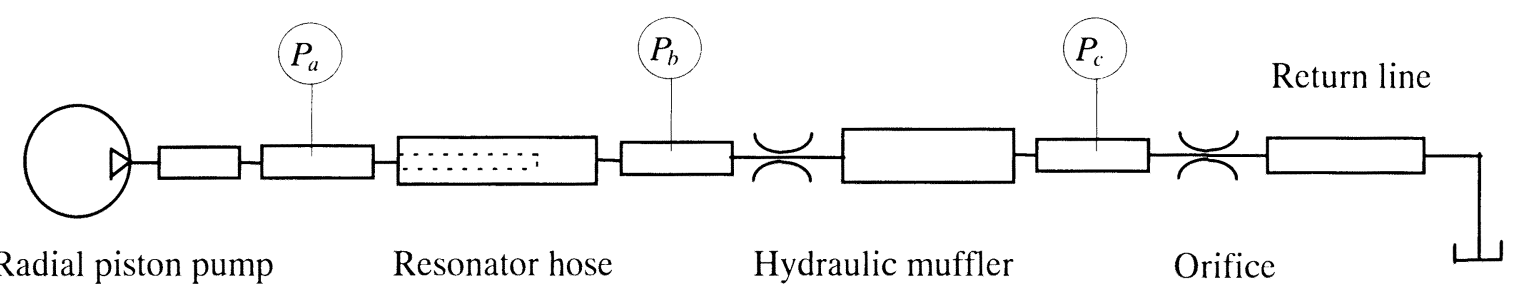

Resorvoir

Figure 5. Schematic of the pump test circuit

In the following sections, we deal with the following two hydraulic circuits;

1) Circuit measuring the transfer characteristics for hydraulic pipelines.

2) A pump-test circuit for the pressure ripple measurement.

For the former circuit, an ideal pressure source model generating sinusoidal pressure is connected to the inlet part of the resonator hose, and the outlet part is terminated by a closed end. The schematic of the circuit model is shown in Figure 4.

For the latter case, the circuit is modelled as shown in Figure 5. A radial piston pump serves an ideal flow source model. A hydraulic silencer is regarded as the combination of a steel pipe and an orifice. For a valve the equation for an orifice is used. A reservoir is regarded as a zero pressure source.

These models are connected to each other in the simulation program PAP, the resonator-hose model being added to it as well. This program can easily simulate any combinations of circuits.

\section{PARAMETER IDENTIFICATION}

The stiffness and damping coefficients of the outer-tube

Table 1. Parameters of a resonator hose

\begin{tabular}{|c|c|}
\hline \multicolumn{2}{|c|}{ Outer tube } \\
\hline$k_{1}$ & $296 \mathrm{E} 9 \mathrm{~N} / \mathrm{m}^{3}$ \\
\hline$k_{2}$ & $358 \mathrm{E} 9 \mathrm{~N} / \mathrm{m}^{3}$ \\
\hline$d$ & $490 \mathrm{E} 6 \mathrm{Ns} / \mathrm{m}^{3}$ \\
\hline \multicolumn{2}{|c|}{ Inner tube } \\
\hline$a_{i}$ & $360 \mathrm{~m} / \mathrm{s}$ \\
\hline$R$ & $2.53 \mathrm{E}-6 \mathrm{~m}^{2} / \mathrm{Pa}^{-2} / \mathrm{s}$ \\
\hline$\alpha$ & 2.46 \\
\hline
\end{tabular}

wall are experimentally estimated using a hose without inner tube[4]. The leakage coefficient and the friction factor of the inner tube are measured by static test. The wave propagation speed in the inner tube is evaluated by measuring resonance frequency. The results are listed in Table 1.

\section{VERIFICATION OF THE SIMULATED RESULTS}

In order to verify the simulation model, the calculated results for the systems are compared to the experimental results.

Figure 6 shows a comparison of the gain $\left(\left|P_{\text {out }} / P_{\text {in }}\right|\right)$ for one resonator hose in the circuit shown in Figure 4 . The first and second resonance frequencies are agree well. The values of the gain are agree fairly well at lower frequencies, but in higher frequencies, they are quite different from each other. This means that the model has a limitation of applicable frequency which ranges from 0

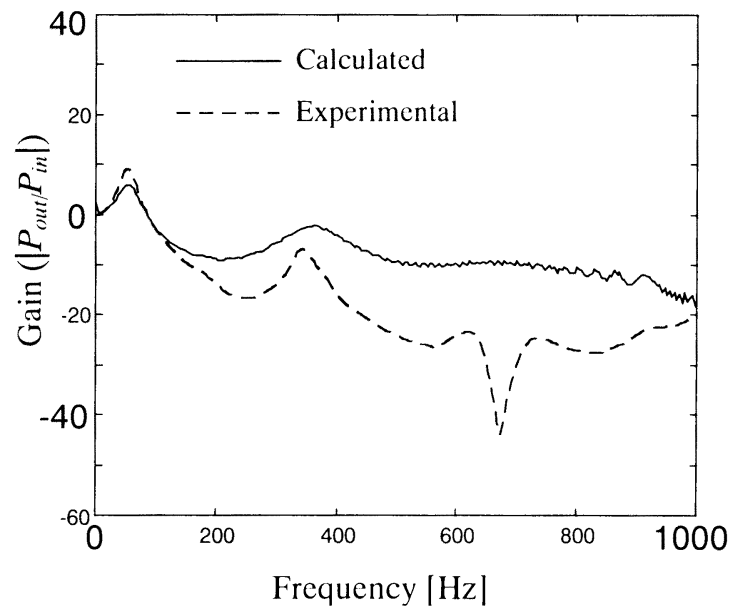

Figure 6. Verification of the model by experiment (Comparison of the transfer gain) 


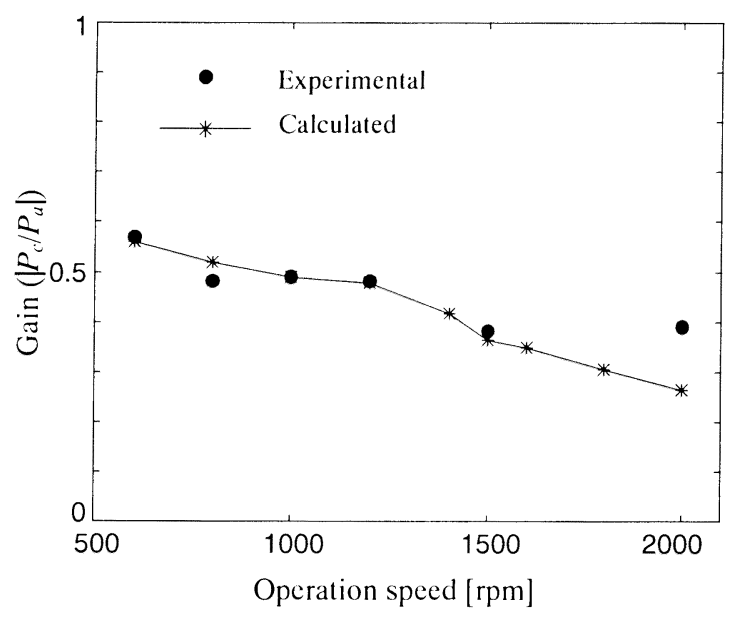

Figure 7. Verification of the model by experiment (Pump test circuit)

to $300 \mathrm{~Hz}$.

Figure 7 shows the comparison of amplitude ratios $\left(P_{c}\right.$ $\left(P_{a}\right)$ for the circuit in Figure 5. The simulated results agree well over the range of $500 \mathrm{rpm}$ to $1500 \mathrm{rpm}$, but at 2000rpm they does not agree with experimental ones. The pump used in this system has 6 pistons, so that it generates flow ripples whose fundamental frequency $f_{1}$ is

$f_{1}=\frac{N z}{60}=\frac{2000 \times 6}{60}=200 \mathrm{~Hz}$

at $2000 \mathrm{rpm}$, where $\mathrm{N}$ is the pump speed and $\mathrm{z}$ is the number of the piston. As seen form the frequency band of the simulation model, it is clear that the model is not satisfactory to calculate in higher pump speeds. However, most of noise problems occur in lower speed range such as an idling of the engine. Then we can use

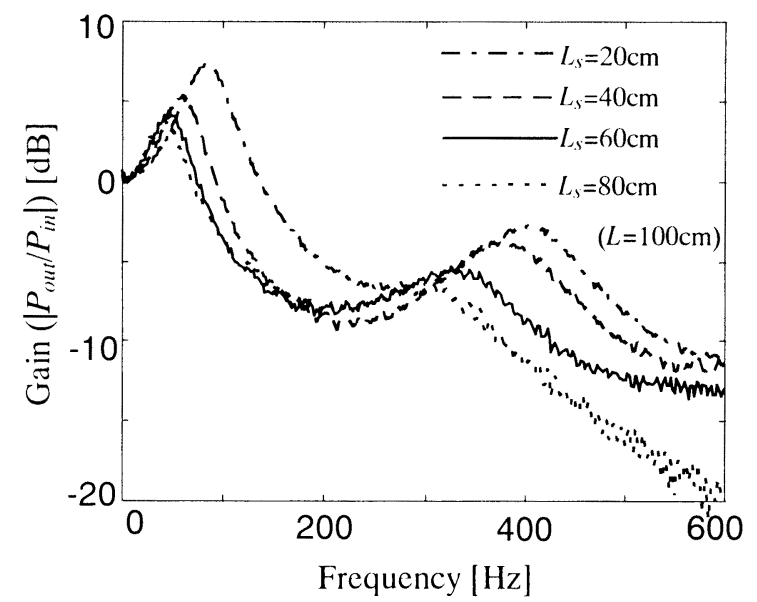

a) Gain of the output pressure for the input pressure the model in the case of slower operation speed. Thus we can conclude that this model is effective in studying noise problems.

\section{PARAMETER STUDY}

Using this simulation model, we shall try to assess the effects of design parameters on the hydrodynamic characteristics of a resonator hose. In the following assessments the length $L$ of the resonator hose is fixed at $1 \mathrm{~m}$ and the inner diameter of the outer tube is also fixed at $0.01 \mathrm{~m}$. The gains of the hoses are calculated for the system shown in Figure 4.

Figures 8 a) and b) show the effect of the length $L_{s}$ of the inner tube on the gains of the transfer matrix. In both of these figures the resonance frequency of the resonator hose decreases with an increase in $L_{s}$. The value of the gain at the resonant frequency decreases also with an increase in $L_{s}$. The trends of the effect of the design parameters on the gain $P_{\text {out }} / P_{\text {in }}$ and $P_{\text {out }} / Q_{\text {in }}$ are almost the same as shown in figures a) and b), then let us assess the effects with the gain of $P_{\text {out }} / P_{\text {in }}$ in the following discussions.

Figure 9 shows the effect of the modification coefficient $\alpha$ in the friction term in the inner tube flow. From this figure we can see that the friction trends to reduce gain and decrease the amplitude at the resonance frequency. Figure 10 shows the effect of the leakage coefficient $R$ of the leakage flow passing through the inner tube wall on gain. The relation between gain and coefficient $R$ is not clear from these results. If we set $R$ to a larger value,

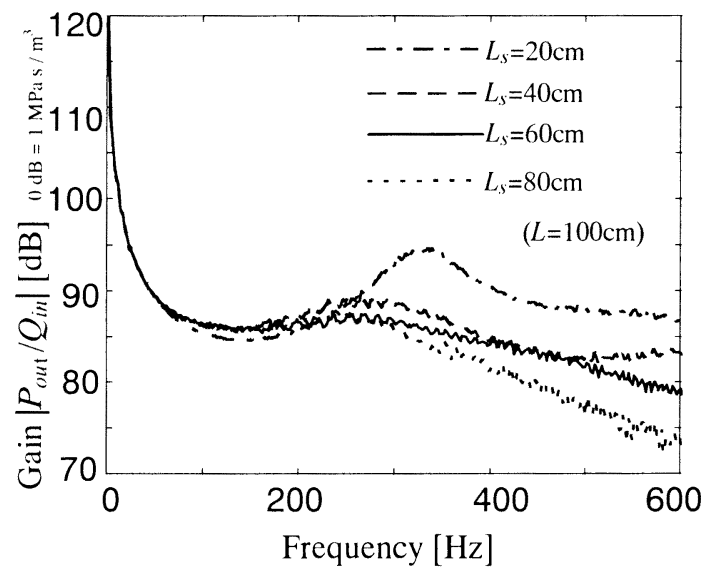

b) Gain of the output pressure for the input flow rate

Figure 8. Effect of the inner-tube length $L_{s}$ 


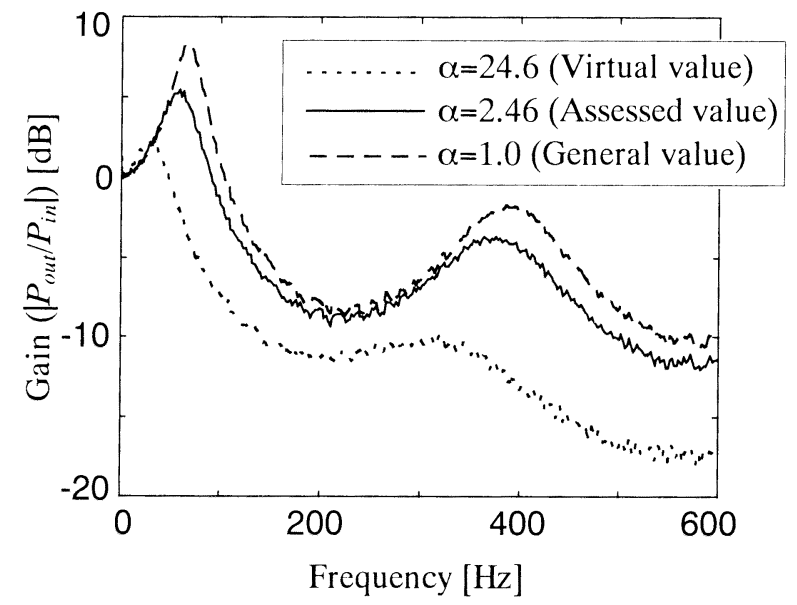

Figure 9. Effect of coefficient $\alpha$

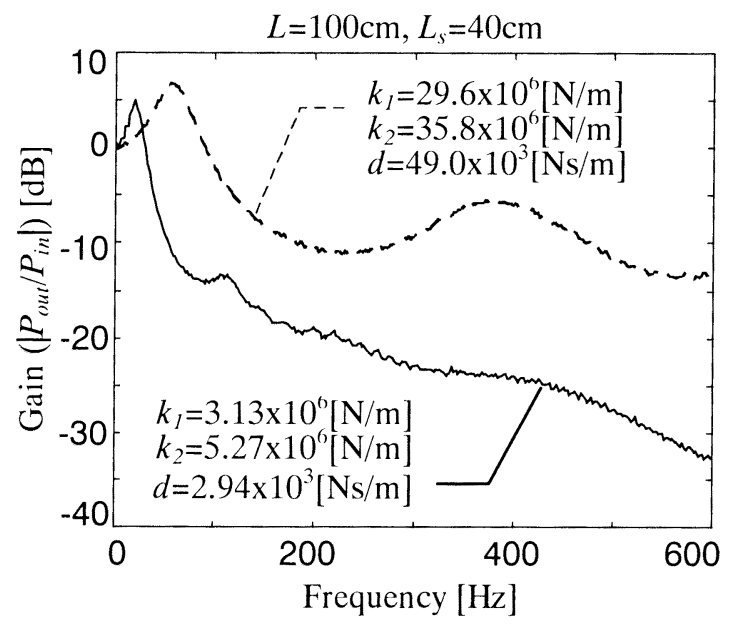

Figure 11. Effect of the stiffness of outer-tube

however, the gain will increase all over the frequency band except the neighborhood of the first resonance frequency. In other words, the leakage has a damping effect on the gain at resonance frequency.

Figure 11 shows the effect of the stiffness of the outertube wall. The soft (low stiffness) wall is very effective to reduce the gain and the resonance frequency.

\section{CONCLUSIONS}

In order to simulate a resonator hose, a simulation model has been developed, using method of characteristics and considering the leakage flow through the inner tube wall. The accuracy of the simulated results were compared with experimental ones, and it is

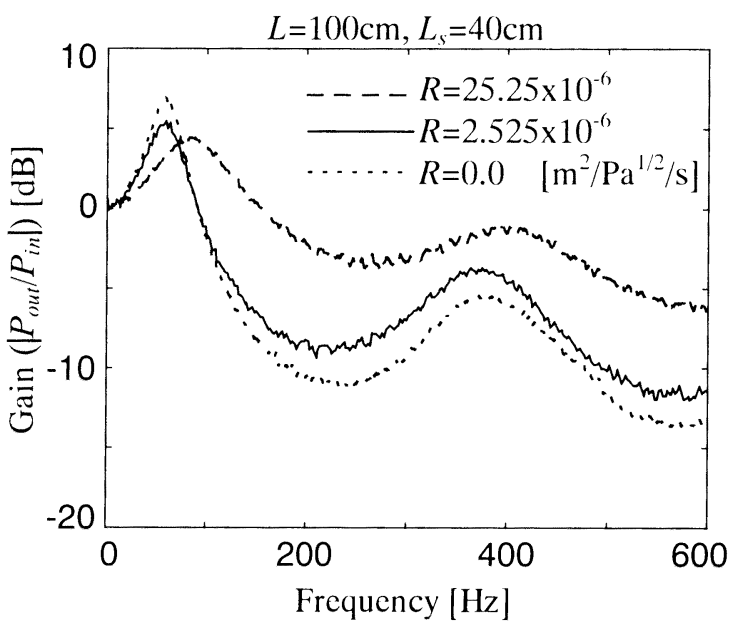

Figure 10. Effect of leakage coefficient $R$

concluded that this simulation model is useful over the frequencies less than $300 \mathrm{~Hz}$.

Using this model, the effect of the following design parameters of resonator hose was investigated:

1) Length of the inner tube,

2) Friction factor of the inner tube,

3) Leakage coefficient of the inner-tube wall, and

4) Stiffness of the outer-tube wall.

As a result, to reduce pressure or flow ripple in a system, it is effective to use soft outer tube, to extend inner tube length, and to increase the restriction of inner tube.

\section{ACKNOWLEDGMENTS}

Authors wish to thank those who supported our study for their assistance and for giving us the permission to publish this work.

\section{REFERENCES}

1. M. C. Hastings and C. C. Chen, Analysis of Tuning Cables for Reduction of Fluid borne Noise in Auto motive Power Steering Hydraulic Lines, SAE paper 931295, 1993

2. K. Hattori, Hydraulic Dumping Characteristics Analysis of A Hose with Spiral Tube, Bulletin of JHPS in spring 1994. (In Japanese)

3. K. Nagata, K. Takahashi, and K. Saitoh, A Simulation Technique for Pressure Fluctuation in a Vane Pump, 8th Bath International Fluid Power Workshop, 1995.

4. A. Kitagawa, A Study on Noise and Vibration Reduction of Fluid Power System, A report of The Japan Society of the Promotion of Machine Industry Technical Research Institute, KSK-GH1-1·1, P207-238, 1990. (In Japanese) 